\title{
Treatment of Extensive Toxic Epidermal Necrolysis in Children
}

\author{
Marcus Spies, MD; Arthur P. Sanford, MD; J. F. Aili Low, MD; Steven E. Wolf, MD; and \\ David N. Herndon, MD
}

\begin{abstract}
Objective. Toxic epidermal necrolysis (TEN) is a rare but life-threatening disease of the skin and mucous membranes. We report our experience in the treatment of pediatric TEN patients with early debridement of necrotic skin and coverage with human allograft skin.

Methods. From 1984 to 2000, 15 children (6 girls, 9 boys, $7.2 \pm 1.5$ years) with a histologic diagnosis of TEN and involvement of $>30 \%$ total body surface area were treated at the Shriners Hospitals for Children in Galveston. All were treated in a specialized pediatric burn intensive care unit after our standard treatment protocol, including operative debridement of sloughing skin and allografting within 24 hours of admission. Outcome parameters were mortality, length of hospital stay, wound healing, clinical complications, causative drugs, corticosteroid use, and delay in referral to a burn center.

Results. Taking a new medication (antibiotics, anticonvulsive drugs) was associated with all cases of TEN. Patients who were treated with early debridement and coverage with allograft skin showed no wound infection, and overall mortality was $7 \%$. Total length of hospital stay was $26 \pm 3$ days. Long-term sequelae were changes in skin pigmentation $(\mathbf{1 0 0 \%})$, ophthalmologic problems $(40 \%)$, and diffuse itching early after wound healing $(53 \%)$.

Conclusion. Although a rare disease in children, TEN was managed successfully in a burn center environment, using early debridement and wound coverage with allograft skin as a biological dressing. The use of corticosteroids and referral patterns seems unchanged during the past 2 decades, indicating an additional need for information and education about the disease. Pediatrics 2001; 108:1162-1168; toxic epidermal necrolysis, children, burn center, mortality, biological dressing.
\end{abstract}

ABBREVIATIONS. TEN, toxic epidermal necrolysis; EM, erythema multiforme minor; SJS, Stevens-Johnson syndrome; TBSA total body surface area; ADEN, acute disseminated epidermal necrosis.

$\mathrm{T}$ oxic epidermal necrolysis (TEN) is a rare but life-threatening exfoliating disease of the skin and mucous membranes. The disease manifests itself by extensive blistering of the skin with full-thickness necrosis of the epidermis and involvement of mucosal surfaces (Fig 1). ${ }^{1-3}$ The skin separa-

From the Shriners Hospitals for Children, Department of Surgery, University of Texas Medical Branch, Galveston, Texas.

Received for publication Apr 9, 2001; accepted Jun 19, 2001.

Reprint requests to (M.S.) Shriners Hospitals for Children, 815 Market St, Galveston, TX 77550-2725. E-mail: maspies@utmb.edu

PEDIATRICS (ISSN 0031 4005). Copyright @ 2001 by the American Academy of Pediatrics. tion occurs at the dermoepidermal junction, and, even in unblistered areas, the epidermis can be removed easily by light tangential pressure (Nikolski's sign).

Erythema multiforme minor (EM), Stevens-Johnson syndrome (SJS), and TEN are a group of severe exfoliative diseases of skin and mucous membranes. There is great controversy on the classification of these exfoliative skin disorders, and the terminology is confusing. Most authors consider SJS and TEN to be the same disease entity, differing only by the area of involved skin. Under this scheme, SJS is considered to affect $<10 \%$ of total body surface area (TBSA), whereas TEN covers $>30 \%$ of TBSA, leaving a zone of overlap between $10 \%$ and $30 \%$ of TBSA, which is referred to as SJS/TEN. ${ }^{1,3-7}$

TEN and SJS both seem to be caused by immunologic reactions to foreign antigens. Drugs are implicated in $77 \%$ to $94 \%$ of cases. ${ }^{8-11}$ Antibacterials and antifungals $(36 \%)$, anticonvulsants $(24 \%)$, analgesics, nonsteroidal anti-inflammatory agents $(38 \%)$, and even corticosteroids (14\%) have been suspected of causing the disease process. ${ }^{11-13}$ The underlying pathophysiologic mechanism is unclear. The histologic characteristics of TEN include dermoepidermal separation resembling a blistering scald burn, followed by necrosis and shedding of the epidermis. ${ }^{14}$ Because of histologic similarities with graft-versushost reaction, specifically infiltration by cytotoxic Tlymphocytes, an autoimmune reaction to keratinocytes has been postulated. ${ }^{1,15}$

The optimal treatment of TEN is controversial. Treatment with systemic corticosteroids has not been shown to improve outcome. ${ }^{5}$ On the contrary, there have been reports of increased mortality with the use of steroids in severe exfoliating cases, as the risk of infectious complications is increased with steroidinduced immunosuppression. ${ }^{16,17}$ Another approach for treatment is the use of immunomodulators such as cyclosporin, cyclophosphamide, or plasmapheresis, ${ }^{3,18}$ which have been successful in cases of TEN. Recently, the administration of intravenous immunoglobulins showed beneficial effects on survival in adult patients. ${ }^{15}$

For pediatric patients and more extensive cases involving $>10 \%$ of TBSA, the current literature recommends transfer to a burn center early in the disease process. ${ }^{4,19,20}$ The general recommendation for treatment of severe cases of TEN is the removal of necrotic epidermis and topical treatment of the wounds. $1,21,22$ Similar to scald burns, the affected areas heal within 2 to 3 weeks if wound infections 


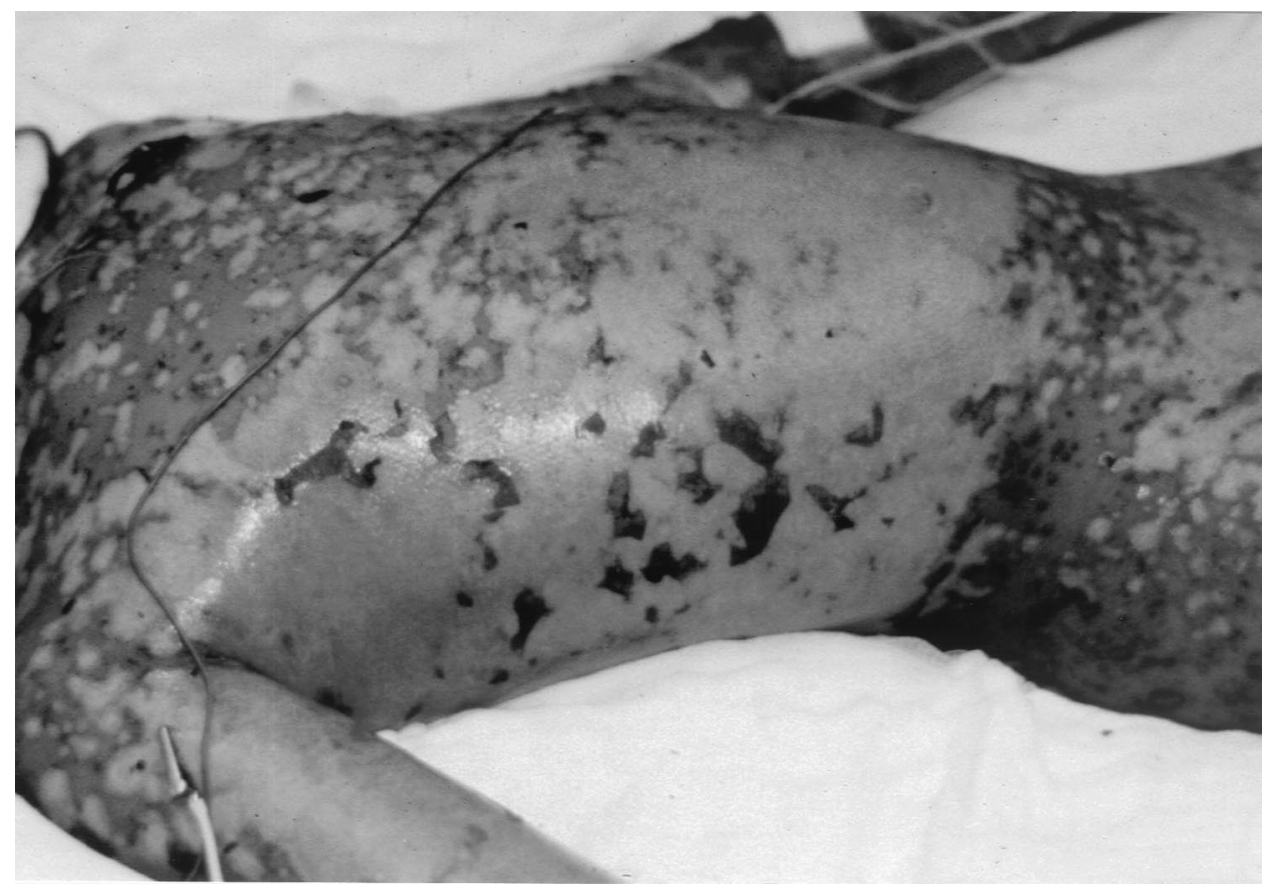

Fig 1. Toxic epidermal necrolysis involving $78 \%$ of TBSA.

can be avoided. To reduce the rate of infections and protect the unhealed areas as well as to reduce pain, several protocols have been suggested. These involve the use of topical antimicrobials such as copper sulfate solution, ${ }^{23}$ silver nitrate, ${ }^{19,24}$ and the coverage of wounds with biological dressings. The most common coverage used is porcine xenograft skin, although there are case reports on the successful use of human cadaveric allograft skin (homograft). The purpose of this study was to report the efficacy of early debridement of necrotic skin and coverage with human allograft skin in the treatment of pediatric TEN patients.

\section{METHODS}

The records of all patients who were treated for exfoliative skin diseases at the Shriners Hospital for Children at Galveston between 1984 and 2000 were reviewed. Fifteen patients with histologically confirmed diagnosis of TEN and involvement of $>30 \%$ of TBSA were found.

\section{Definition of TEN}

We defined patients as experiencing TEN if they had large confluent blisters with Nikolski's sign present, the TBSA involved was $>30 \%$, and the histology of blistering areas showed a fullthickness necrosis of the epidermis and separation at the dermoepidermal junction. ${ }^{15,16,25-27}$

\section{Treatment}

All patients were treated in a specialized pediatric burn intensive care unit at the Shriners Hospital for Children in Galveston after our standard treatment protocol. This included debridement of sloughing skin under operating room conditions within 24 hours after admission. All detached epidermis and all blisters were removed under general anesthesia. With endotracheal intubation for anesthesia, bronchoscopy was performed to determine the extent of respiratory tract involvement. Before mechanical debridement, the whole body was washed with polyvidone-iodine soap. After removal of all necrotic epidermis, all open areas were covered with human allograft skin (meshed 2:1) and a finemesh gauze dressing impregnated with polymyxin/nystatin ointment. The cadaveric allograft was obtained from an American
Association of Tissue Banks-accredited skin bank (Skin Bank, Shriners Hospital for Children or Skin Bank, University of Texas Medical Branch, Galveston). In the study, 2 patients each were covered with xenograft skin (porcine) at a referring institution or with Biobrane (Bertek, Morgantown, WV) at our center as human allograft skin was temporarily unavailable. Identical to the treatment of severely burned patients, dressings were changed daily with the polymyxin/nystatin ointment application. Adherent allograft was not removed, but sites with nonadherent grafts were regrafted within 7 days. Local infection of wounds was monitored by microbiologic testing 2 times a week. Wound infection was defined by local purulent secretion and positive microbiologic testing.

In cases of corticosteroid treatment begun before admission to our institution, these were tapered off rapidly. All patients were seen and followed throughout hospital course by an ophthalmologist. Continuous attention was given to eye care, including lubrication with ointment every 2 hours and application of topical erythromycin every 6 hours. Eyelid synechias were broken gently by the ophthalmologist as required. All patients received antacids, oral nystatin, and intensive respiratory care.

All patients received fluid resuscitation with lactated Ringer's solution. The amount was calculated depending on the extent of epidermal loss following the SBH-Galveston formula for pediatric burns $\left(5000 \mathrm{~mL} / \mathrm{m}^{2}\right.$ body surface area burned $/ \mathrm{d}+2000 \mathrm{~mL} / \mathrm{m}^{2}$ TBSA/d) and adjusted to clinical parameters.

Enteral feeding via nasoduodenal tube with Vivonex T.E.N. (Novartis, East Hanover, NJ) was started within the 24 hours of admission. The dose was adjusted to reach daily caloric requirements as estimated by the modified Harris-Benedict formula (1800 $\mathrm{kcal} / 24 \mathrm{~h}$ per TBSA plus $1300 \mathrm{kcal} / 24 \mathrm{~h}$ per TBSA affected for children aged 1-12 years and $1500 \mathrm{kcal} / 24 \mathrm{~h}$ per TBSA plus 1500 $\mathrm{kcal} / 24 \mathrm{~h}$ per TBSA affected for patients older than 12 years).

We started a Benadryl (diphenhydramine, Parke-Davis, Morris Plains, NJ) drip infusion at $1 \mathrm{mg} / \mathrm{kg} / \mathrm{h}$ on admission in an attempt to halt the histaminergic response. This was continued until wound healing was completed and patients were near hospital discharge. All patients were treated with broad-spectrum antibiotics on admission. The antibiotics were adjusted on the basis of microbiology cultures to inhibit growth of identified organisms. Antibiotics were chosen to avoid those with a known higher risk for the development of TEN (eg, trimethoprim/sulfamethoxazole, penicillins)

The hospital course and outcome was assessed for all patients, and a comparison was made between the results of different coverage materials. Use of corticosteroids in the referring hospital 
and referral patterns were compared for time periods before and after January 1992. All data are presented as mean \pm standard error of the mean. Statistical analysis was by unpaired $t$ test or $\chi^{2}$ test where applicable.

\section{RESULTS}

\section{Patient Demographics}

Fifteen children (6 girls, 9 boys) aged $7.2 \pm 1.5$ years with a histologic diagnosis of TEN and an involvement of $>30 \%$ of TBSA were identified. Seven (47\%) were black, 7 (47\%) were white, and 1 (7\%) was Hispanic. The mean size of the involved surface area was $76 \% \pm 5 \%$ of TBSA.

\section{Hospital Course}

All children had fever $\left(>39^{\circ} \mathrm{C}\right)$ associated with the onset of the disease. Nine children (60\%) showed clinical involvement of the respiratory tract, resulting in persistent productive cough, sore throat, and mucosal sloughing of the pharyngeal region. However, on bronchoscopy, only 2 patients (13\%) showed mucosal involvement of trachea and the bronchial tree. One child with involvement of the respiratory tract died 5 days after TEN onset; the other child required prolonged mechanical ventilation for 51 days. A total of 7 children (47\%) required ventilatory support for an average of $11 \pm 8$ days.

Six children $(40 \%)$ showed involvement of the gastrointestinal tract in the form of vomiting and diarrhea, but only $1(7 \%)$ presented with gastrointestinal blood loss. All but 1 (93\%) had ocular (conjunctival) involvement. None of the children were positive for the human immunodeficiency virus.

With the exception of the 1 child who required prolonged ventilation, all surviving children had a short and uncomplicated hospital course with undisturbed wound healing. The mean total length of hospital stay was $26 \pm 3$ days.

\section{Medications}

In all children, a new medication had been started shortly before the onset of the symptoms. The mean duration of medication until the first symptoms of TEN (blistering, fever) occurred was $11 \pm 2$ days. In 11 children $(73 \%)$, TEN occurred after starting antibiotics. Four of these children received concurrent anticonvulsive medication (phenobarbital). Table 1 shows the medications and the indication for their prescription. Only 2 patients had a known drug allergy (1 to sulfonamides, 1 to penicillin) before the disease onset.

\section{Corticosteroid Use}

Systemic corticosteroids were administered in 7 of 8 children who were admitted before January 1992, compared with 4 of 7 who were admitted after this time $\left(P=1.0, \chi^{2}\right.$ test $)$. Corticosteroids often were administered at the initial time point of disease manifestation in referring institutions and continued for $5 \pm 2$ and $2 \pm 1$ days, respectively.

\section{Referral to Burn Center}

Before 1992, referral to the specialized burn center took place an average of $8 \pm 2$ days after disease
TABLE 1. Drug Exposure and Reasons for Administration

\begin{tabular}{lcl}
\hline $\begin{array}{c}\text { Reason for } \\
\text { Administration }\end{array}$ & $\begin{array}{c}\text { Number of } \\
\text { Children }\end{array}$ \\
\hline Fever & 1 & \multicolumn{1}{c}{ Medication* } \\
& 3 & $\begin{array}{l}\text { Trimethoprim/sulfamethoxazole } \\
\text { Amoxicilin }\end{array}$ \\
& 1 & $\begin{array}{l}\text { Cephalosporins } \\
\text { Ibuprofen }\end{array}$ \\
Otitis media & 2 & Trimethoprim/sulfamethoxazole \\
Enterococcal sepsis in & 1 & Trimethoprim/sulfamethoxazole \\
30\% TBSA burn & & \\
Upper airway & 1 & Trimethoprim/sulfamethoxazole \\
infection & 1 & Erythromycin \\
& 1 & Trimethoprim/sulfamethoxazole \\
Acne & 1 & Tetracycline \\
Conjunctivitis & 1 & Amoxicillin \\
Seizures & 4 & Phenobarbital \\
Severe headaches & 1 & Phenytoin \\
& 1 & Chlordia-zepoxide and \\
\end{tabular}

* Some patients were exposed to more than 1 of these drugs.

onset. After January 1992, children were admitted to our institution $5 \pm 1$ days after disease onset $(P=.19$, $t$ test).

\section{Treatment}

All patients were taken to the operating room within 1 day of admission. After cleansing and superficial debridement of the blistering areas, 11 children $(73 \%)$ were treated with allograft skin coverage. Two children $(13 \%)$, initially seen at other institutions, were covered with porcine xenograft skin, and 2 children $(13 \%)$ were covered with Biobrane synthetic dressing. Four of the children covered with allograft were taken to the operating room a second time for removal and replacement of nonadherent grafts within an average of 5 days. The allograft skin then adhered to the wounds in all cases and served as a temporary partially incorporated biological dressing (Fig 2). The allograft skin then gradually separated from the healing wounds after 2 to 3 weeks and was removed without discomfort for the patient.

Both of the children who were treated with Biobrane developed local purulent wound infection with bacterial overgrowth that required removal of the dressing material. These children then underwent daily dressing changes and bathing and were alternatively covered with dressings that were soaked in hypochlorite and impregnated with polymyxin/mycosporin ointment. No progression of the infection and development of full-thickness injury could be observed. Children who were treated with human allograft or porcine xenograft showed no signs of wound infection.

\section{Mortality}

The only death that occurred in our pediatric patients with TEN was in a 9-month old infant. The male patient was admitted to the Shriners Hospitals for Children with a 30\% TBSA scald burn, which itself is a potentially lethal injury in this age group. During a 20-day hospital course, he developed sepsis attributable to a trimethoprimsulfamethoxazole-sensitive enterococcus strain. In 


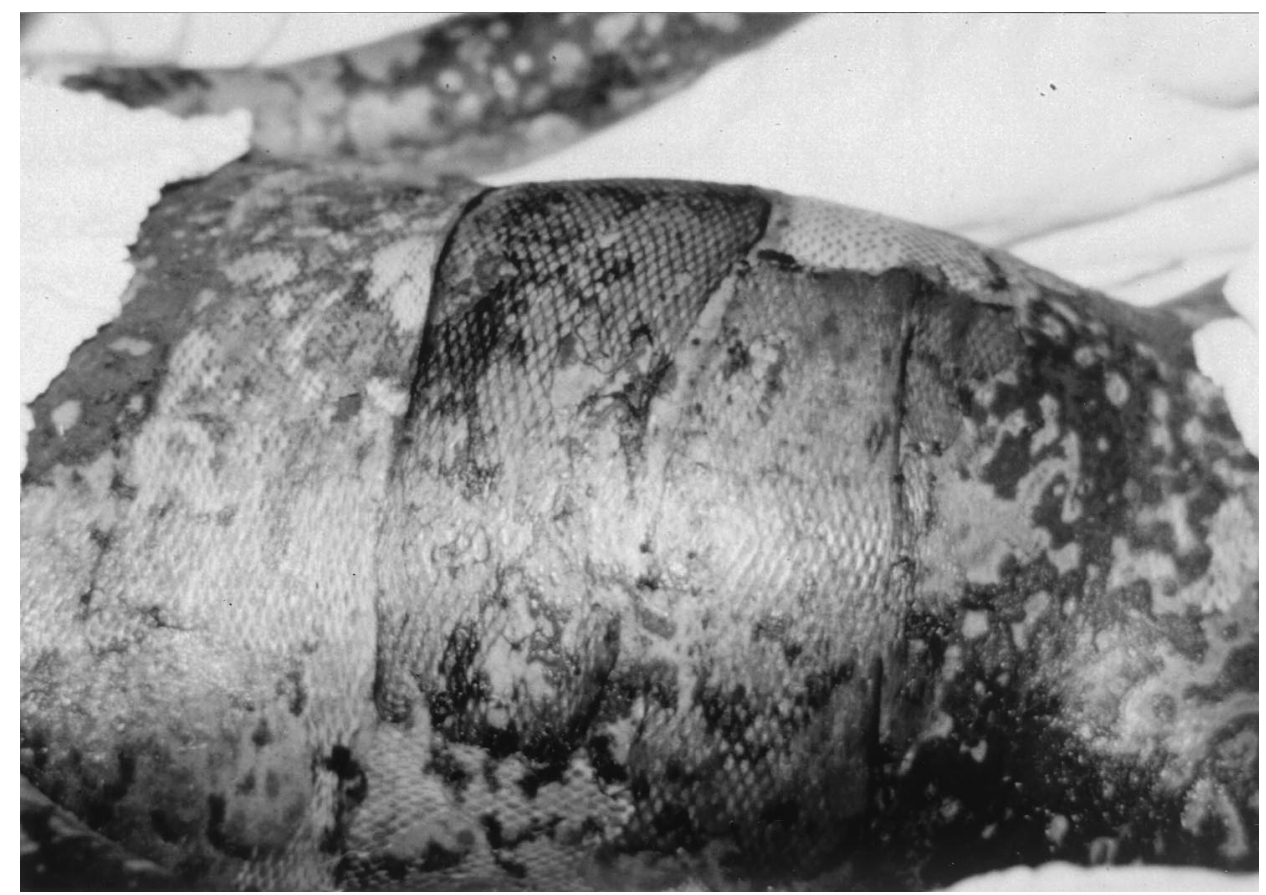

Fig 2. The same patient after debridement and application of allograft skin.

addition to established broad-spectrum antibiotic medication, he received a single dose of trimethoprimsulfamethoxazole. After this single dose administration of trimethoprim-sulfamethoxazole, a progressive blistering of the integument occurred with total loss of $73 \%$ of epidermal surface. Within a day of onset of the first TEN symptoms, the patient's debridement and coverage with human allograft skin was performed in the operating room. Subsequently, multiorgan dysfunction and death by cardiac arrest occurred 5 days after the onset of TEN.

\section{Long-Term Sequelae}

Eleven children were followed up to 12 months. None of these patients developed scarring, but all patients had residual patchy hyper- or hypopigmented areas (Fig 3). Hypopigmentation was seen predominantly in black children, and hyperpigmentation was seen in white children. This dyspigmentation decreased over time. Six patients $(40 \%)$ reported persistent ophthalmological problems with photophobia, chronic conjunctivitis sicca, conjunctival synechias, or recurrent lacrimal cysts requiring operative intervention. Eight of the patients (53\%) had diffuse itching in the early phase after wound healing, which in all cases was treated with antihistamines.

\section{DISCUSSION}

TEN is a rare disease in adults and even more rare in children. Here, we report on 15 pediatric patients with extensive TEN with a mean involved body surface area of $76 \%$. These patients were treated at the Shriners Hospitals for Children in Galveston over a period of 15 years. This is one of the largest series of pediatric patients reported with TEN. An additional interesting fact is the ethnic distribution of our patients with a relatively high number of black children
(47\%) presenting at our institution. If this is attributable to the referral mechanism to the Shriners Hospital system, the socioeconomic situation or a genetic predisposition is difficult to evaluate given the low

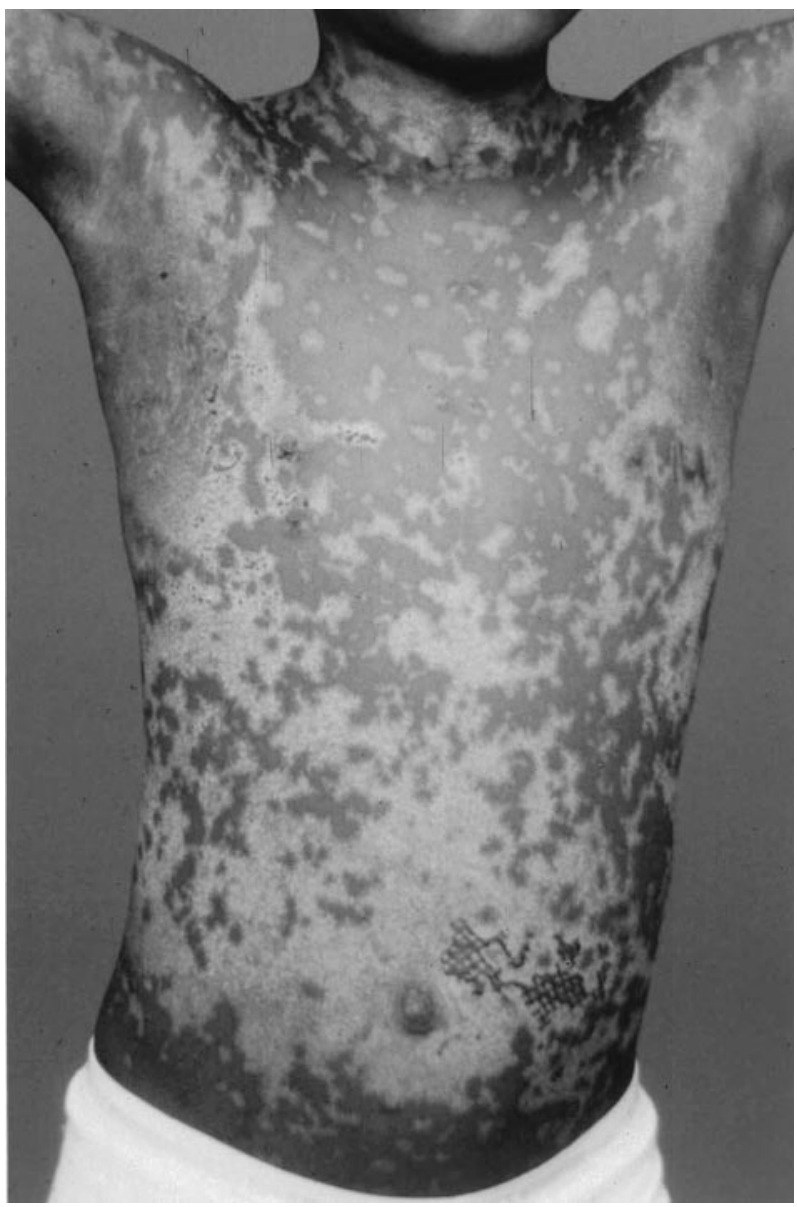

Fig 3. Hypopigmentation of involved skin areas after 1 year. 
incidence of the disease and the lack of a central registry for TEN in the United States.

The mortality in our patient series was low $(7 \%)$. The only death in the 15 children occurred in an infant. The 9-month-old developed sepsis after a 30\% TBSA scald burn. He received a single-dose administration of trimethoprim/sulfamethoxazole for this condition, which was followed by manifestation of TEN involving 73\% TBSA with development of multiorgan dysfunction; death by cardiac arrest occurred 5 days after onset of the disease.

In review, the disease most likely was triggered by the administration of specific drugs, mainly sulfonamide antibiotics, $\beta$-lactam antibiotics, and anticonvulsive drugs. The use of corticosteroids and the time from diagnosis to referral to an adequate treatment center was not decreased during the studied period. Meticulous wound care with early debridement of necrotic tissue and the use of human cadaveric allograft as a biological dressing material enabled rapid closure of open wounds. Common longterm complications seen in our patient population were skin dyspigmentation and ocular sequelae, such as conjunctivitis sicca or conjunctival synechias.

TEN is a rare disease. The incidence in adults is estimated to be between 0.4 and 1.2 cases per 1 million people per year. The incidence of SJS has been reported to be 1 to 7 cases per 1 million people per year. ${ }^{8-10,25,28}$ In a study on patients who were treated at Group Health Cooperative in Seattle from 1972 to 1986, an incidence of 0.5 cases per 1 million people per year was found for TEN alone. ${ }^{9}$ In a study in Germany from 1990 to 1992, the calculated incidence of TEN and SJS together was 1.89 per 1 million people per year. ${ }^{12}$ These exfoliative disorders occur in all age groups; however, the incidence is increased in the elderly and in females. $13,8,24$ The reported incidence of TEN in children is lower than in adults. ${ }^{2,3}$ The evaluation of treatment and outcome in children is therefore difficult and based on small study populations. In recent literature, TEN has a rate of mortality of up to $60 \%$, mainly attributable to septic complications ${ }^{17,19,20,26,29,30}$ Death often occurs early in the course of the disease with sepsis being the most frequent cause. Pseudomonas aeruginosa and Staphylococcus aureus are the predominant organisms involved. ${ }^{30}$ Mortality is increased significantly in pa- tients who are at the extremes of age and in relation to the percentage of denuded skin. 1,21,23,24,26

In review of the literature, most publications only present low numbers of pediatric TEN patients (Table 2). Ruiz-Maldonado ${ }^{23}$ reported on a series of 60 children who were treated at the Department of Dermatology of the National Institute of Pediatrics in Mexico City with severe exfoliating disease. He suggested a new classification, the acute disseminated epidermal necrosis (ADEN) type 1-3. Applying his definitions, our patients would be classified as ADEN types 2 and 3 . When compared with his data, only 21 patients presented had a disease severity similar to our patient population. In this study, the mortality was $37.5 \%$ for children with an involvement of $60 \%$ to $70 \%$ of TBSA (ADEN type 2 ) and $60 \%$ for those with an involvement of $>70 \%$ of TBSA (ADEN type 3), which is significantly higher than our mortality of $7 \%$. Two other studies with large patient numbers by Murphy et $\mathrm{al}^{20}$ and Revuz et $\mathrm{al}^{6}$ reported on 44 and 87 cases of TEN including patients aged from 0.8 to 90 years and 2 to 90 years, respectively. Unfortunately, in these studies, no information is given on the number of involved children. Prendiville et $\mathrm{al}^{30}$ published the successful treatment of 7 pediatric patients with TEN and 14 with SJS in a pediatric hospital without any mortality. However, a comparison is problematic, as this publication does not quantify the TBSA involved in a way similar to other publications. Sheridan et al ${ }^{31}$ reported complete survival of all of their 10 pediatric patients using a treatment concept similar to ours with early cleansing and removal of necrotic epithelium and coverage with predominately porcine xenograft. The described patient group basically matched ours in age and extent of disease. Considering the currently available data and the treatment modalities, a mortality below $15 \%$ seems to be an achievable goal.

The actual pathophysiologic mechanism of TEN remains debated. However, it seems apparent that an exposure to certain drugs plays a significant role in triggering the disease response. In our study group, antibacterial agents, mostly sulfonamide and $\beta$-lactam antibiotics, and phenobarbital were the main culprit drugs. This is in accordance with studies performed by Roujeau et $\mathrm{al}^{8,11,13}$ and Schoepf

TABLE 2. Review of Literature

\begin{tabular}{|c|c|c|c|c|}
\hline Authors (Year) & $\begin{array}{l}\text { Number of } \\
\text { Patients }\end{array}$ & $\begin{array}{l}\text { Pediatric } \\
\text { Patients }\end{array}$ & $\begin{array}{c}\text { Involvement } \\
(\% \text { TBSA) }\end{array}$ & $\begin{array}{c}\text { Mortality } \\
(\%)\end{array}$ \\
\hline Adzick et al $(1985)^{19}$ & 4 & 4 & $86 \pm 6$ & 25 \\
\hline Ruiz-Maldonado $(1985)^{23}$ & 5 & 5 & $>70$ & 60 \\
\hline Ruiz-Maldonado $(1985)^{23}$ & 16 & 16 & $60-70$ & 37.5 \\
\hline Heimbach et al (1987) & 19 & 3 & $75 \pm 5$ & 16 \\
\hline Revuz et al $(1987)^{6}$ & 87 & $\mathrm{~N} / \mathrm{A}$ & $39 \pm 3$ & 25 \\
\hline Jones et al $(1989)^{17}$ & 9 & 9 & $60 \pm 6.5$ & 11 \\
\hline Taylor et al (1989) 21 & 6 & 6 & $72.5 \pm 7$ & 17 \\
\hline Prendiville et al $(1989)^{30}$ & 7 & 7 & N/A & 0 \\
\hline Murphy et al (1997) $)^{20}$ & 44 & $\mathrm{~N} / \mathrm{A}$ & $52 \pm 5$ & 36 \\
\hline McGee et al $(1998)^{27}$ & 36 & $\mathrm{~N} / \mathrm{A}$ & $63 \pm 5$ & 31 \\
\hline Sheridan et al (1999) 31 & 10 & 10 & $76 \pm 6$ & 0 \\
\hline Spies et al (2001) & 15 & 15 & $76 \pm 5$ & 7 \\
\hline
\end{tabular}

Data are means \pm standard error of the mean. N/A indicates not available. 
et $\mathrm{al}^{25}$ using the databases of the French-European and the German central registry for severe skin reactions. These studies demonstrated an increased risk with administration of trimethoprim-sulfamethoxazole and other sulfonamide antibiotics, aminopenicillins, quinolones, and cephalosporins. Increased risks also could be seen for anticonvulsants, such as phenobarbital, phenytoin, carbamazepine, and valproic acid; anti-inflammatory drugs; acetaminophen; allopurinol; and corticosteroids. Some of the mentioned drugs are prescribed to infants, toddlers, and older children frequently. Others, such as antipyretics and analgesics, are readily available over the counter. It is interesting that corticosteroid use also is related to an increased incidence of TEN, which may serve as an additional argument against systemic corticosteroid administration. The current literature is opposed to the use of steroids. ${ }^{21,24,30} \mathrm{In}$ several studies, the use of steroids was not beneficial; on the contrary, many authors discussed a higher risk of infection and thus increased mortality with steroid administration. 5,17,20,24 When a treatment with corticosteroids had been started before transfer to our hospital, it was tapered off rapidly. When comparing patients who were admitted before and after January 1992, no significant difference in corticosteroid use in the referring hospitals could be found. This highlights that additional education and information on diagnosis and therapy of this severe drug-related disease are necessary.

In our patient group, the only TEN manifestation that occurred in our hospital was triggered by trimethoprim-sulfamethoxazole. This definitely demonstrated that the use of certain medications, such as trimethoprim-sulfamethoxazole, needs increased awareness of the risk-benefit relation. As most of our patients did not have any medical history of sensitization to drugs, antibiotic agents should be administered cautiously. The indication of antibiotics has to be verified constantly to avoid this kind of adverse drug reaction.

Another interesting fact in the evaluation of our patient group is that the delay until a definite diagnosis is made and the patient is transferred to a burn center has not significantly decreased over the past 2 decades. However, this is a main focus of the pertinent literature, as burn centers are considered the most appropriate treatment facilities. Some authors claimed that the early transfer of the patient significantly improves survival and outcome. ${ }^{20,21,27}$

Infections and sepsis are the main causes of death in TEN patients. ${ }^{17,20,26,27}$ Therefore, all efforts should be made to reduce the risk of infections to improve survival. The administration of prophylactic antibiotics is highly controversial. However, during the study period, it was standard operating procedure in our burn center. Most authors tend to use systemic antibiotics specifically once localized bacterial colonization or infection has occurred. ${ }^{16,30,31}$ However, from the burn surgeon's point of view, the prevention of wound infection and wound sepsis is of utmost importance to improve chances of survival in this disease.

The optimal treatment of the blistering skin is a major issue discussed in the literature. Heimbach et $\mathrm{al}^{22}$ treated 19 patients, including 3 children, by debridement and wound coverage with porcine xenograft. They stated that the prevention of infection and the protection of the wound surface are of great importance for fast and undisturbed skin regeneration. Alternatively, Adzick et $\mathrm{al}^{19}$ treated 4 children with TEN on a burn unit using $0.5 \%$ silver nitrate soaks and dressing changes every 12 hours. Biological wound coverages such as allograft or xenograft were not used, and, therefore, open observation of the wounds was not possible. In their report, 1 single death occurred as a result of aspiration pneumonia. Prendiville et $\mathrm{al}^{30}$ removed only spontaneously blistering skin and did not recommend additional debridement. The group based this recommendation on the fact that the skin reepithelializes under intact blisters. Open areas were covered with topical antimicrobial agents or synthetic hydrogel. An even less aggressive approach was used by Jones et al. ${ }^{17}$ They debrided only necrotic areas and ruptured blisters followed by covering the wounds with wet dressings without use of topical antimicrobials. Their experience shows no differences in the rate of infection or healing time to previous patients treated with topical antimicrobials. In common, all authors emphasize the importance of treating TEN patients in facilities that are specialized to care for patients with extensive skin loss. However, as shown by Prendiville et $\mathrm{al}^{30}$ children with TEN also can be treated successfully in a pediatric intensive care unit. Also in this setting, important conditions for success are meticulous wound care and the prevention of all systemic and local infections.

As for adjuvant administration of systemic corticosteroids, other adjuvant approaches remain under scrutiny until enough clear evidence of benefit is available. This includes the use of immunomodulators such as cyclosporin, cyclophosphamide, or plasmapheresis, ${ }^{3,18}$ which have been used successfully in cases of TEN with the rationale of eliminating causing immune mechanisms or precipitating mediators. Also, administration of mediator-neutralizing effectors of keratinocyte apoptosis in the form of intravenous immunoglobulins has shown beneficial effects on survival in adult patients. ${ }^{15}$ However, as mentioned earlier, most studies of this particular disease lack the evidence of prospective studies that are based on large patient groups.

Our presented standard treatment includes the removal of all loose skin and coverage of the wound with banked allograft skin. In our view, the aggressive debridement of blisters and the necrotic epidermis removes the site of disease induction and concurrently reduces the risk of local infection. The epidermal layer in the blister roof is necrotic and therefore does not supply reliable protection for the underlying dermis. The necrotic tissue itself can be a source for infection. Coverage of the wound with biological dressing material as homologous skin grafts provides optimal protection until the epidermis completely regenerates. With this strategy, wound infections are rare and virtually no scarring occurs. Clinically, the use of biological dressing ma- 
terial on the openly exposed dermis and the sensible dermal nerve endings seems to minimize immensely the pain and physical discomfort of the patients. In addition, frequent and often painful dressing changes can be avoided. Most topical agents used, such as silver nitrate, decrease keratinocyte and fibroblast proliferation and thus impede wound healing and reepithelialization. The allograft skin provides the optimal wound coverage as it is partially incorporated and later rejected with increasing reepithelialization. Xenograft does not incorporate and therefore offers reduced protection. Similarly, Biobrane initially may adhere well to the wounds; however, in our experience, the coverage of large body areas resulted in extended wound infection. In both of our cases, the Biobrane had to be totally removed and the wounds treated conservatively.

The relatively large percentage $(40 \%)$ of ocular long-term complication is disturbing, as it severely affects the patient's quality of life and his or her functional abilities. Sheridan et $\mathrm{al}^{31}$ reported a $20 \%$ incidence of conjunctivitis sicca in 10 patients, but this apparently does not include a long-term followup. It needs to be emphasized that during acute treatment, all efforts should be undertaken to prevent ocular sequelae. This must include the daily assessment and treatment by an experienced ophthalmologist. Other complications, however, such as hypo- or hyperpigmentation, mostly irritating to the patient and his or her environment, are of lesser importance. It is interesting that hypopigmented skin areas appear in all black children, whereas focal hyperpigmentation is more likely to occur in white patients. Hyperpigmented areas also tend to fade over time. In the report by Sheridan et $\mathrm{al}^{31}$ skin pigmentation changes occurred in 50\%; however, there is no information on the involved ethnic skin types.

\section{CONCLUSION}

Our treatment strategy seems to be successful and safe in the treatment of pediatric TEN patients. This includes the strict avoidance of corticosteroid administration. Prevention of local wound infection is of utmost importance and can be obtained by early debridement of the necrotic epidermis and all blisters and coverage with human allograft skin as a biological dressing. In a long-term perspective, however, additional information and education are necessary to establish a more critical use of known culprit drugs and better communication with specialized centers to improve referral patterns. Furthermore, a central national registry would be helpful to ease research and increase the scarce knowledge regarding this disease.

\section{REFERENCES}

1. Avakian R, Flowers FP, Araujo OE, Ramos-Caro FA. Toxic epidermal necrolysis: a review. J Am Acad Dermatol. 1991;25:69-79

2. Roujeau JC, Chosidow O, Saiag P, Guillaume JC. Toxic epidermal necrolysis (Lyell syndrome). J Am Acad Dermatol. 1990;23:1039-1058

3. Becker DS. Toxic epidermal necrolysis. Lancet. 1998;351:1417-1420

4. Rasmussen JE. Erythema multiforme. Should anyone care about the standards of care? Arch Dermatol. 1995;131:726-729
5. Smoot EC III. Treatment issues in the care of patients with toxic epidermal necrolysis. Burns. 1999;25:439-442

6. Revuz J, Roujeau JC, Guillaume JC, Penso D, Touraine R. Treatment of toxic epidermal necrolysis. Creteil's experience. Arch Dermatol. 1987;123: 1156-1158

7. Goldstein SM, Wintroub BW, Elias PM, Wuepper KD. Toxic epidermal necrolysis. Unmuddying the waters. Arch Dermatol. 1987;123:1153-1156

8. Roujeau JC, Guillaume JC, Fabre JP, Penso D, Flechet ML, Girre JP. Toxic epidermal necrolysis (Lyell syndrome). Incidence and drug etiology in France, 1981-1985. Arch Dermatol. 1990:126:37-42

9. Chan HL, Stern RS, Arndt KA, et al. The incidence of erythema multiforme, Stevens-Johnson syndrome, and toxic epidermal necrolysis. A population-based study with particular reference to reactions caused by drugs among outpatients. Arch Dermatol. 1990;126:43-47

10. Strom BL, Carson JL, Halpern AC, et al. A population-based study of Stevens-Johnson syndrome. Incidence and antecedent drug exposures. Arch Dermatol. 1991;127:831-838

11. Guillaume JC, Roujeau JC, Revuz J, Penso D, Touraine R. The culprit drugs in 87 cases of toxic epidermal necrolysis (Lyell's syndrome). Arch Dermatol. 1987;123:1166-1170

12. Rzany B, Mockenhaupt M, Baur S, et al. Epidemiology of erythema exudativum multiforme majus, Stevens-Johnson syndrome, and toxic epidermal necrolysis in Germany (1990-1992): structure and results of a population-based registry. J Clin Epidemiol. 1996;49:769-773

13. Roujeau JC, Kelly JP, Naldi L, et al. Medication use and the risk of Stevens-Johnson syndrome or toxic epidermal necrolysis. N Engl J Med. 1995;333:1600-1607

14. Lyell A. Toxic epidermal necrolysis: an eruption resembling scalding of the skin. Br J Dermatol. 1956;68:355-361

15. Viard I, Wehrli P, Bullani R, et al. Inhibition of toxic epidermal necrolysis by blockade of CD95 with human intravenous immunoglobulin. Science. 1998;282:490-493

16. Halebian PH, Corder VJ, Madden MR, Finkelstein JL, Shires GT. Improved burn center survival of patients with toxic epidermal necrolysis managed without corticosteroids. Ann Surg. 1986;204:503-512

17. Jones WG, Halebian P, Madden M, Finkelstein J, Goodwin CW. Druginduced toxic epidermal necrolysis in children. J Pediatr Surg. 1989;24: 167-170

18. Spies M, Hollyoak M, Muller MJ, Goodwin CW, Herndon DN. Exfoliative and necrotizing diseases of the skin. In: Herndon DN, ed. Total Burn Care. 2nd ed. Philadelphia, PA: WB Saunders; 2001:492-501

19. Adzick NS, Kim SH, Bondoc CC, Quinby WC, Remensnyder JP. Management of toxic epidermal necrolysis in a pediatric burn center. Am J Dis Child. 1985;139:499-502

20. Murphy JT, Purdue GF, Hunt JL. Toxic epidermal necrolysis. J Burn Care Rehabil. 1997;18:417-420

21. Taylor JA, Grube B, Heimbach DM, Bergman AB. Toxic epidermal necrolysis. A comprehensive approach. Multidisciplinary management in a burn center. Clin Pediatr (Phila). 1989;28:404-407

22. Heimbach DM, Engrav LH, Marvin JA, Harnar TJ, Grube BJ. Toxic epidermal necrolysis. A step forward in treatment. JAMA. 1987;257: 2171-2175

23. Ruiz-Maldonado R. Acute disseminated epidermal necrosis types 1, 2, and 3: study of sixty cases. J Am Acad Dermatol. 1985;13:623-635

24. Halebian P, Corder VJ, Herndon DN, Shires GT. A burn center experience with toxic epidermal necrolysis. J Burn Care Rehabil. 1983;4:176-183

25. Schopf E, Stuhmer A, Rzany B, Victor N, Zentgraf R, Kapp JF. Toxic epidermal necrolysis and Stevens-Johnson syndrome. An epidemiologic study from West Germany. Arch Dermatol. 1991;127:839-842

26. Revuz J, Penso D, Roujeau JC, et al. Toxic epidermal necrolysis. Clinical findings and prognosis factors in 87 patients. Arch Dermatol. 1987;123: 1160-1165

27. McGee T, Munster A. Toxic epidermal necrolysis syndrome: mortality rate reduced with early referral to regional burn center. Plast Reconstr Surg. 1998;102:1018-1022

28. Naldi L, Locati F, Marchesi L, Cainelli T. Incidence of toxic epidermal necrolysis in Italy. Arch Dermatol. 1990;126:1103-1104

29. Scully MC, Frieden IJ. Toxic epidermal necrolysis in early infancy. J Am Acad Dermatol. 1992;27:340-344

30. Prendiville JS, Hebert AA, Greenwald MJ, Esterly NB. Management of Stevens-Johnson syndrome and toxic epidermal necrolysis in children I Pediatr. 1989;115:881-887

31. Sheridan RL, Weber JM, Schulz JT, Ryan CM, Low HM, Thompkins RG. Management of severe toxic epidermal necrolysis in children. I Burn Care Rehabil. 1999;20:497-500 